\title{
Déterminisme de la prolifération des cyanobactéries toxiques en Côte d'Ivoire
}

\author{
Julien COULIBALY-KALPY ${ }^{1,2 *}$, Man-Koumba SOUMAHORO ${ }^{1}$, \\ Julie Estelle NIAMIEN-EBROTTIE ${ }^{3}$, Kadjowely YEO ${ }^{1}$, Lydie AMON ${ }^{2}$, \\ Alico Joseph DJAMAN ${ }^{1,2}$ et Mireille DOSSO ${ }^{1,2}$ \\ ${ }^{1}$ Institut Pasteur de Côte d'Ivoire; 01 BP 490 Abidjan 01. \\ ${ }^{2}$ Université Félix Houphouët-Boigny ; BPV 34 Abidjan, Côte d'Ivoire. \\ ${ }^{3}$ Université Nangui-Abrogoua ; 02 BP 801 Abidjan 02, Côte d'Ivoire. \\ *Auteur correspondant,_E-mail :jc_kalpy@yahoo.fr; Tel : +22507915176.
}

\section{RESUME}

Les blooms de cyanobactéries surviennent dans les eaux de surface, en particulier dans les eaux où les conditions physiques et les charges en éléments nutritifs sont favorables à leur prolifération. L'objectif de ce travail a été de déterminer les principaux paramètres physico-chimiques favorisant l'efflorescence des cyanobactéries dans le Sud-Est de la Côte d'Ivoire. La distribution des cyanobactéries et le déterminisme de leur développement ont été étudiés de juin à octobre sur la lagune Aghien, les retenues d'Adzopé et d'Agboville. Les paramètres physico-chimiques ont été mesurés et la composition ainsi que la densité des cyanobactéries ont été réalisés. Le niveau de turbidité, la température, la quantité de phosphates, la quantité de nitrite et la quantité de phosphore sur les sites sont les facteurs déterminants l'abondance des cyanobactéries sur les sites. Un maximum de $39,4 \times 10^{6}$ cellules/ $\mathrm{ml}$ a été rencontré au mois d'octobre. Les genres dominants sont Microcystis (40\%), Anabaena (plus de 14\%), Aphanocapsa (10\%) et Oscillatoria (8\%) dans les milieux étudiés. Ce sont en effet des genres potentiellement toxiques. De plus, pendant la période d'échantillonnage des blooms à Microcystis en juin, les associations Microcystis-Planktothrix en juillet et Microcystis-Anabeana en septembre, ont été observés. La prolifération des cyanobactéries dans les plans d'eau étudiés est sous le contrôle de l'hypereutrophisation à travers la concentration en nutriment mais aussi des paramètres physiques. (C) 2017 International Formulae Group. All rights reserved.

Mots clés : Cyanobactéries, lagune Aghien, déterminisme, prolifération, Côte d'Ivoire.

\section{Determination of the proliferation of toxic cyanobacteria in Côte d'Ivoire}

\begin{abstract}
Cyanobacteria blooms occur in surface waters, especially in waters where physical conditions and nutrient loads are favourable for their proliferation. The aim of this work was to determine the main physicochemical parameters favoring the efflorescence of the cyanobacteria in the South-East of Côte d'Ivoire. The distribution of cyanobacteria and the determinism of their development were studied from June to October on the Aghien lagoon, the Adzopé and Agboville reservoirs. Physico-chemical parameters were measured and also the composition and density of the cyanobacteria. The level of turbidity, temperature, quantity of
\end{abstract}


phosphates, amount of nitrite and amount of phosphorus are factors determining the abundance of cyanobacteria at the sites. A maximum of $39.4 \times 10^{6}$ cells $/ \mathrm{ml}$ was encountered in October. The dominant genera are Microcystis (40\%), Anabaena (more than 14\%), Aphanocapsa (10\%) and Oscillatoria (8\%) in the studied area. These are indeed potentially toxic genera. In addition, during sampling period, blooms of Microcystis in June, the Microcystis-Planktothrix associations in July and Microcystis-Anabeana in September were observed. The proliferation of cyanobacteria in the studied water bodies is under the control of hypereutrophisation through the nutrient concentration but also physical parameters.

(C) 2017 International Formulae Group. All rights reserved.

Keywords: Cyanobacteria, Aghien lagoon, determinism, proliferation, Côte d'Ivoire.

\section{INTRODUCTION}

La qualité des eaux de surface, un enjeu majeur pour l'avenir de notre planète, est de plus en plus altéré partout dans le monde par l'eutrophisation (Ni et al., 2012). Cette dernière crée les conditions pour une prolifération du phytoplancton en générale et des cyanobactéries en particulier (Ni et al., 2012). Les cyanobactéries sont des procaryotes à Gram négatif, photosynthétiques qui sont également appelées cyanophycées, cyanophytes ou algues bleues (Garrity et al., 2001). Les proliférations de cyanobactéries ont des conséquences multiples à la fois sur le fonctionnement des écosystèmes aquatiques et sur leurs usages. Les cyanobactéries sont capables de produire des métabolites secondaires dont les conséquences économiques peuvent être importantes dans les piscicultures (Robin et al., 2006). Un grand nombre d'espèces de cyanobactéries est capable de synthétiser diverses toxines qui peuvent avoir des impacts néfastes sur la faune et la flore aquatiques, la santé des animaux terrestres (Briand et al., 2008) et de l'Homme (Fosso-Kankeu et al., 2008). Les " blooms ", " fleurs d'eau » ou encore « efflorescences» dominés par les cyanobactéries sont, pour plus de la moitié toxiques (Maršálek et al., 2000). A ce jour, de nombreux cas de mortalités animales liés aux efflorescences de cyanobactéries toxiques ont déjà été signalés en Belgique et dans le monde entier (Carmichael et al., 2001; NiamienEbrottié et al., 2015). Ces développements massifs de cyanobactéries potentiellement toxiques constituent donc un réel danger pour la population. Aussi ont-ils été reconnus comme un problème émergent de santé publique à la fin du $20^{\text {ème }}$ siècle surtout pour les populations dépendant des eaux de surfaces pour leur adduction en eaux potables (Ouellette et Wilhelm, 2003 ; Te et al., 2011). En effet, il n'est pas rare de constater des proliférations de cyanobactéries toxiques dans les réservoirs utilisés pour la production d'eau potable (Chorus et al., 2000). Ainsi, plusieurs cas d'intoxication (allergies, diarrhées, vomissements) (Bertrand et al., 2004) et de mortalité humaine ont été rapportés notamment au Brésil (Azevedo et al., 2002; Willame et al., 2005).

En Afrique, la limitation des eaux souterraines, l'accroissement exponentiel de la démographie et l'urbanisation accélérée ont poussé les Etats à fournir d'énormes efforts pour mobiliser les eaux de surface afin de fournir de l'eau potable aux populations.

En Côte d'Ivoire, de nombreux plans d'eau de surface sont déjà utilisés pour la production d'eau potable (Etien N'dah et al., 1996). Face à l'épuisement de la nappe dite du plateau continental alimentant la ville d'Abidjan, la lagune Aghien pourrait permettre de pallier aux déficits déjà récurrents observés dans la métropole ivoirienne. Cependant la présence de cyanobactérie et l'eutrophisation de nombreux plan d'eau (N'gohesse et al., 2007 ; Adon et al., 2011) pourraient constituer un danger en cas de prolifération massive. La compréhension et la prédiction de l'apparition d'efflorescence de cyanobactéries toxiques pourrait être un avantage pour les gestionnaires et les utilisateurs des plans d'eau. L'objectif de cette étude est de faire 
l'inventaire des cyanobactéries potentiellement toxiques dans les réservoirs d'eau potable d'une part et d'autre part identifier le déterminisme de leur développement.

\section{MATERIEL ET METHODES}

\section{Choix des stations d'échantillonnage}

Le choix des stations d'échantillonnage s'est fait sur la base de travaux antérieurs pour certains sites: stations sur la lagune Aghien selon les travaux de Humbert (2012) sur le phytoplancton, stations de la retenue d'Adzopé (Adon et al., 2013). La station d'échantillonnage d'Agboville a été le point de prélèvement de l'eau par la station de traitement d'eau. Ainsi, 11 stations ont été retenues sur la Lagune Aghien, 3 sur la Retenue d'Adzopé, 1 sur le celle d'Agboville.

\section{Méthode d'échantillonnage}

Les échantillons ont été prélevés dans différents flacons et conservés selon le type d'analyse à effectuer.

A chaque station, 20 litres d'eau ont été prélevés à environ 0,5 mètre de profondeur et filtrée avec un filet à plancton de $20 \mu \mathrm{m}$ de maille. Le culot retenu a été transvasé dans un tube de Falcon et a été fixé au lugol à $1 \% \mathrm{v} / \mathrm{v}$ ou au formladéhyde à $5 \%$ pour l'identification morphologique des cyanobactéries.

Des échantillons de $50 \mathrm{~mL}$ ont été prélevés et fixés comme indiqué ci-dessus dans des bouteilles stériles. Ces derniers ont servi pour le dénombrement des cyanobactéries.

\section{Identification et dénombrement}

Concernant l'identification, les échantillons fixés ont été homogénéisés manuellement puis $50 \mu \mathrm{L}$ ont été prélevés et observés entre lame et lamelle au microscope optique, au grossissement X100, puis X400. Cette identification est réalisée grâce aux caractères morphologiques des cyanobactéries décris par Ouattara et al. (2000), John et al. (2003); Leitao et Couté (2005), Komárek et Anagnostidis $(1998 ; 2005)$. Des photos ont été réalisées à l'aide d'un appareil photo numérique Nikon COOLPIX P500. Le dénombrement a permis de déterminer la concentration en cyanobactéries aux différentes stations. Il s'est fait en utilisant une cellule de comptage hématimétrique de type cellule de Malassez.

\section{Mesure des paramètres physico-chimique}

Les mesures de la température, de la conductivité, de la turbidité et du pH ont été effectuées in situ entre 9 et 10 heures du matin. Les éléments nutritifs à savoir, le nitrate, le nitrite, le phosphore, le phosphate, le fer total ont été mesurés au laboratoire par photométrie. Les pastilles réactives correspondant à chaque paramètre ont été utilisées en suivant la procédure donnée par le fabricant. Les concentrations de phosphore ont permis de classifier les plans d'eau en utilisant les Recommandations canadiennes pour la qualité des eaux (CCME, 2004).

\section{Analyses statistiques des données}

Les données ont été recueillies sur le terrain à partir d'un cahier d'observation conçu pour l'étude. Les paramètres ont été décrits par leurs moyennes assorties de leurs valeurs maximales et minimales. Le test de corrélation de Pearson a été utilisé après standardisation des moyennes des différents paramètres pour étudier les relations entre eux. Les données ont été introduites et conservées dans le logiciel Epidata 3.1 puis analysés à l'aide du logiciel d'analyse Stata11.0.

Afin de mettre en évidence les paramètres influençant la prolifération des cyanobactéries, une analyse de redondance (RDA) a été effectuée à l'aide du logiciel Canoco 5 après avoir vérifié le test de permutation de Monte Carlo au seuil de 0.05 .

\section{RESULTATS}

\section{Paramètres physico-chimiques \\ Paramètres physiques}

La conductivité moyenne sur l'ensemble des sites était de 60,17 $\mu \mathrm{S} / \mathrm{cm}$ (min : 28,8 $\mu \mathrm{S} / \mathrm{cm} ; \max : 230 \mu \mathrm{S} / \mathrm{cm}$ ). La valeur la plus faible a été enregistrée à Aghien 4 et la plus élevée à Aghien 1. En moyenne la 
conductivité était de $67 \mu \mathrm{S} / \mathrm{cm}(\mathrm{min}=28,8$; $\max =230 \mu \mathrm{S} / \mathrm{cm}$ ) sur la lagune Aghien, de $42,36 \mu \mathrm{S} / \mathrm{cm} \quad(\min =29,50 ; \quad \max =59,70$ $\mu \mathrm{S} / \mathrm{cm})$ au niveau du seuil (retenue) d'Agboville et de 41,01 $(\mathrm{min}=36,6 \mu \mathrm{S} / \mathrm{cm}$; $\max =54 \mu \mathrm{S} / \mathrm{cm})$ à Adzopé.

La turbidité a été en moyenne de 48,71 NTU sur l'ensemble des sites $(\mathrm{min}=6,98$; $\max =165$ NTU). Sur la lagune Aghien, la turbidité moyenne était de 56,34 NTU ( $\mathrm{min}=$ $6,98 \mathrm{NTU} ; \max =165 \mathrm{NTU}$ ). Les valeurs de la turbidité sur le seuil (retenue) d'Agboville étaient de 16,66 NTU en moyenne ( $\mathrm{min}=$ $10,09 \mathrm{NTU} ; \max =28,40 \mathrm{NTU}$ ). La valeur moyenne de la turbidité à Adzopé était de 31,40 NTU.

La température a été en moyenne de $27,27{ }^{\circ} \mathrm{C}$ pour l'ensemble des sites. La température la plus faible $\left(25^{\circ} \mathrm{C}\right)$ a été enregistrée à Adzopé 3 et à Agboville alors que la plus élevée $\left(29,60^{\circ} \mathrm{C}\right)$ l'a été à Adzopé 1. Pour le site d'Aghien, la température moyenne a été de $27,30{ }^{\circ} \mathrm{C}\left(\mathrm{min}=25,60{ }^{\circ} \mathrm{C}\right.$; $\left.\max =29,20^{\circ} \mathrm{C}\right)$. En moyenne, la température était de $26,70{ }^{\circ} \mathrm{C}$ à Agboville $\left(\min =25{ }^{\circ} \mathrm{C}\right.$; $\left.\max =29,1{ }^{\circ} \mathrm{C}\right)$. Il a été enregistré une température moyenne de $27,38^{\circ} \mathrm{C}$ à Adzopé $\left(\min =25^{\circ} \mathrm{C} ; \max =29,60^{\circ} \mathrm{C}\right)$

Le $\mathrm{pH}$ moyen a été de 7,22 pour l'ensemble des sites avec un minimum de 5,20 à Aghien 5 (Station Aghien 5) au mois de juillet et un maximum à 8,70 à la Aghien 7 (station Aghien 7) au mois d'octobre.

Au niveau de la Lagune Aghien, le $\mathrm{pH}$ a varié de 5,20 à 8,70 . Le $\mathrm{pH}$ oscillait entre 7,47 et 8,34 ) au niveau du seuil d'Agboville et entre 6,59 et 8,41 à Adzopé.

\section{Paramètres chimiques}

La valeur moyenne du Phosphore dans la retenue d'eau d'Adzopé était de $0,15 \mathrm{mg} / \mathrm{L}$ $(\min =0,074 \mathrm{mg} / \mathrm{L} ; \max =0,31 \mathrm{mg} / \mathrm{L})$. Quant aux concentrations de phosphate, elles ont varié entre un minimum de 0,23 et un maximum de $0,96 \mathrm{mg} / \mathrm{L}$ avec une moyenne de $0,44 \mathrm{mg} / \mathrm{L}$. La concentration moyenne en Nitrate était de $2,56 \mathrm{mg} / \mathrm{L}$ avec des extrémités allant de 0,20 à $4,60 \mathrm{mg} / \mathrm{L}$. Le nitrite avait pour concentration moyenne $0,03 \mathrm{mg} / \mathrm{L}$ avec un minimum de 0,01 et un maximum de 0,06
mg/L. (Tableau 1). A Agboville, le phosphore a été en moyenne de $0,15 \mathrm{mg} / \mathrm{L}$ avec des valeurs comprises entre 0,09 et $0,21 \mathrm{mg} / \mathrm{L}$. La valeur la plus faible du phosphore a été dosée en Août. Le phosphate a enregistré sa plus faible concentration $(0,29 \mathrm{mg} / \mathrm{L})$ en Août également et la plus forte $(0,66 \mathrm{mg} / \mathrm{L})$ en Octobre. La concentration moyenne de Phosphate était de $0,48 \quad \mathrm{mg} / \mathrm{L}$. La concentration moyenne de Nitrate a été de $3,39 \mathrm{mg} / \mathrm{L}$ avec des valeurs oscillant entre 0,76 et $6,2 \mathrm{mg} / \mathrm{L}$. Les nitrites ont eu une concentration maximale de $0,04 \mathrm{mg} / \mathrm{L}$ et minimal de $0 \mathrm{mg} / \mathrm{L}$ pour une moyenne de 0,01 $\mathrm{mg} / \mathrm{L}$ (Tableau 1). La valeur moyenne $\mathrm{du}$ Phosphore dans la Lagune Aghien était de $0,23 \mathrm{mg} / \mathrm{L}(\mathrm{min}=0,06 ; 0,56 \mathrm{mg} / \mathrm{L})$ quand les concentrations de phosphate ont varié entre un minimum de 0,04 et un maximum de $0,22 \mathrm{mg}$ $/ \mathrm{L}$ avec une moyenne de $0,01 \mathrm{mg} / \mathrm{L}$. Concernant les nitrates et les nitrites, il a été observé des concentrations moyennes respectives de $1,39 \mathrm{mg} / \mathrm{L} \quad(\min =0,003$; $\max =2,94 \mathrm{mg} / \mathrm{L})$ et $0,59 \mathrm{~m} / \mathrm{L} \quad(\min =0,0011$; $\max =3,43 \mathrm{mg} / \mathrm{L}$ ).

\section{Variation spatio-temporelle des abondances de Cyanobactéries \\ Variation temporelle}

$\mathrm{Au}$ cours de la présente étude, la biomasse totale de cyanobactérie a été étudiée et estimée afin de suivre son évolution au cours de l'étude (Figure 1). Une baisse de la charge totale en cyanobactéries a été observée de juin à juillet. Par la suite, les proliférations successives ont permis d'atteindre un maximum de $39,4 \times 10^{6}$ cellules $/ \mathrm{ml}$ au mois d'octobre à la fin de la période de prélèvement.

\section{Proportion des cyanobactéries}

Dans l'ensemble, le genre Microcystis (40\%) était dominant, suivi des genres Anabaena (plus de 14\%), Aphanocapsa (10\%) et Oscillatoria (8\%). Le genre Planktothrix, a représenté environ $3 \%$ des cyanobactéries rencontrées au cours de la présente étude (Figure 2).

Les taxons des genres potentiellement toxique tel que Aphanocapsa, Microcystis, 
Oscillatoria, Planktothrix ont été observés pendant toute l'étude indépendamment du site. Il en est de même pour le genre Chroococcus.

\section{Variation spatiale}

Les proportions des différents genres et espèces de cyanobactéries ont varié tout au long de l'étude. Au niveau de la Lagune Aghien, les principaux genres de cyanobactéries observés étaient composés par ordre d'importance de Microcystis (58\%), Anabeana (16\%), Aphanocapsa (12\%) et Oscillatoria (6\%). Le genre Planktothrix était également présent mais de façon moins importante avec $2 \%$ de la biomasse totale. (Tableau 2). La retenue d'Adzopé a mis en évidence une dominance du genre Oscillatoria avec $32 \%$ des cyanobactéries dénombrées, suivi de Microcystis (20\%) et de Planktothrix $(16 \%)$. Les genres précédemment cité sont tous potentiellement toxiques. Cependant, un genre non toxique Chroococcus a été retrouvé avec une proportion de $16 \%$ de la quantité de cyanobactéries dénombrées (Tableau 3). Le site d'Agboville était lui dominé par Oscillatoria $(90 \%$ de la biomasse de cyanobactérie) et Anabeana (6\%) (Tableau 4). Trois blooms sur la Lagune Aghien et un bloom sur la retenue d'Adzopé ont été observés au cours de la présente étude. Sur la Lagune Aghien, le bloom était dominé par Microcystis en juin, les associations Microcystis-Planktothrix en juillet et Microcystis-Anabeana en septembre. Cependant, au niveau de la retenue d'Adzopé, la prolifération était dominée par Microcystis avec quelques Pseudoanabeana.

Tout le long de cette étude, une augmentation du nombre de cyanobactéries en générale a été observée.

\section{Analyse des corrélations entre les paramètres environnement et l'évolution des cyanobactéries. \\ Analyses univariées}

Le niveau de turbidité, la température, la quantité de phosphates, la quantité de nitrite et la quantité de phosphore sur les sites sont les facteurs déterminants de la présence ou de l'absence de micro-organismes sur un site. Le test de Pearson a montré une forte corrélation positive significative entre l'abondance de cyanobactéries et le nitrite $(r=0,99$; $\mathrm{p}<0,017)$. Cette corrélation positive concerne le site d'Agboville et d'Adzopé mais pas la Lagune Aghien. Au niveau de la Lagune Aghien, il a été montré une corrélation positive entre le nombre de genres de cyanobactéries et la turbidité $(\mathrm{r}=0,67$; $\mathrm{p}<0,02$ ). Il est à noter une association moyennement forte mais négative entre les nitrates et les Anabeana $(r=0,72 ; p<0,01)$.

Les résultats de l'analyse RDA (Redundancy Analysis) indiquent que les deux premiers axes expriment la plus grande variabilité soit $87.2 \%$. Cette ordination indique que la température, le phosphore, le phosphate, le nitrate, le nitrite, la turbidité et le $\mathrm{pH}$ sont les variables environnementales les plus importants qui affectent la distribution des cyanobactéries. Le nitrite est corrélé positivement à l'axe 1 . Les valeurs de nitrate sont élevées aux stations de la lagune Aghien au mois d'octobre et influence la distribution de Anabaena circinalis, Phormidium sp, Anabaena sp, Pseudoanabaena sp, Microcystis wesenbergii, Microcystis aeruginosa et Microcystis sp. Négativement à l'axe 1 sont corrélés le phosphore, le phosphate, le nitrate et la turbidité. Ces paramètres sont associés aux abondances de Cylidrospermopsis sp et Synechoccus sp aux mois de juin et juillet aux stations de la lagune Aghien et d'Adzopé. La température est corrélée positivement à l'axe 2 et affectent l'abondance de Planktothrix sp et Anabaenopsis sp, aux stations S8 et S10 de la lagune Aghien en juin et juillet. Quant au pH, il est corrélé négativement à l'axe 2 et agit sur l'abondance de Chroococcus sp, Aphanocapsa sp et Aphanocapsa incerta aux mois de septembre à la station $\mathrm{S} 11$ et au mois d'août aux stations S4 et S8 dans la lagune Aghien et en juin à la station 3 d'Adzopé. 
Tableau 1: Concentration moyenne des nutriments dans les plans d'eaux étudiés.

\begin{tabular}{ccccccc}
\hline & & Juin 2014 & Juillet 2014 & Août 2014 & Septembre 2014 & Octobre 2014 \\
\hline \multirow{4}{*}{ Adzopé } & Phosphore (mg/L) & 0,18 & 0,14 & 0,10 & 0,10 & 0,22 \\
& Phosphate (mg/L) & 0,57 & 0,44 & 0,31 & 0,35 & 0,59 \\
& Nitrite (mg/L) & 13930,58 & 13940,53 & 0,20 & 13961,15 & 13971,27 \\
& Nitrate (mg/L) & 4643,78 & 4647,03 & 0,20 & 4653,87 & 4657,36 \\
& Phosphore (mg/L) & 0,11 & 0,19 & 0,09 & 0,17 & 0,21 \\
\multirow{3}{*}{ Agboville } & Phosphate (mg/L) & 0,32 & 0,59 & 0,29 & 0,53 & 0,66 \\
& Nitrite (mg/L) & 0,00 & 0,01 & nd & 0,00 & 0,04 \\
& Nitrate (mg/L) & 0,76 & 6,20 & 4,20 & 0,80 & 5,00 \\
& Phosphore (mg/L) & 0,28 & 0,36 & 0,14 & 0,24 & 0,14 \\
& Phosphate (mg/L) & 0,05 & 0,15 & 0,08 & 0,07 & 0,05 \\
& Nitrite (mg/L) & 0,04 & 0,18 & 0,04 & 0,01 & 2,60 \\
& Nitrate (mg/L) & 0,72 & 2,37 & 1,20 & 2,25 & 0,17 \\
\hline
\end{tabular}

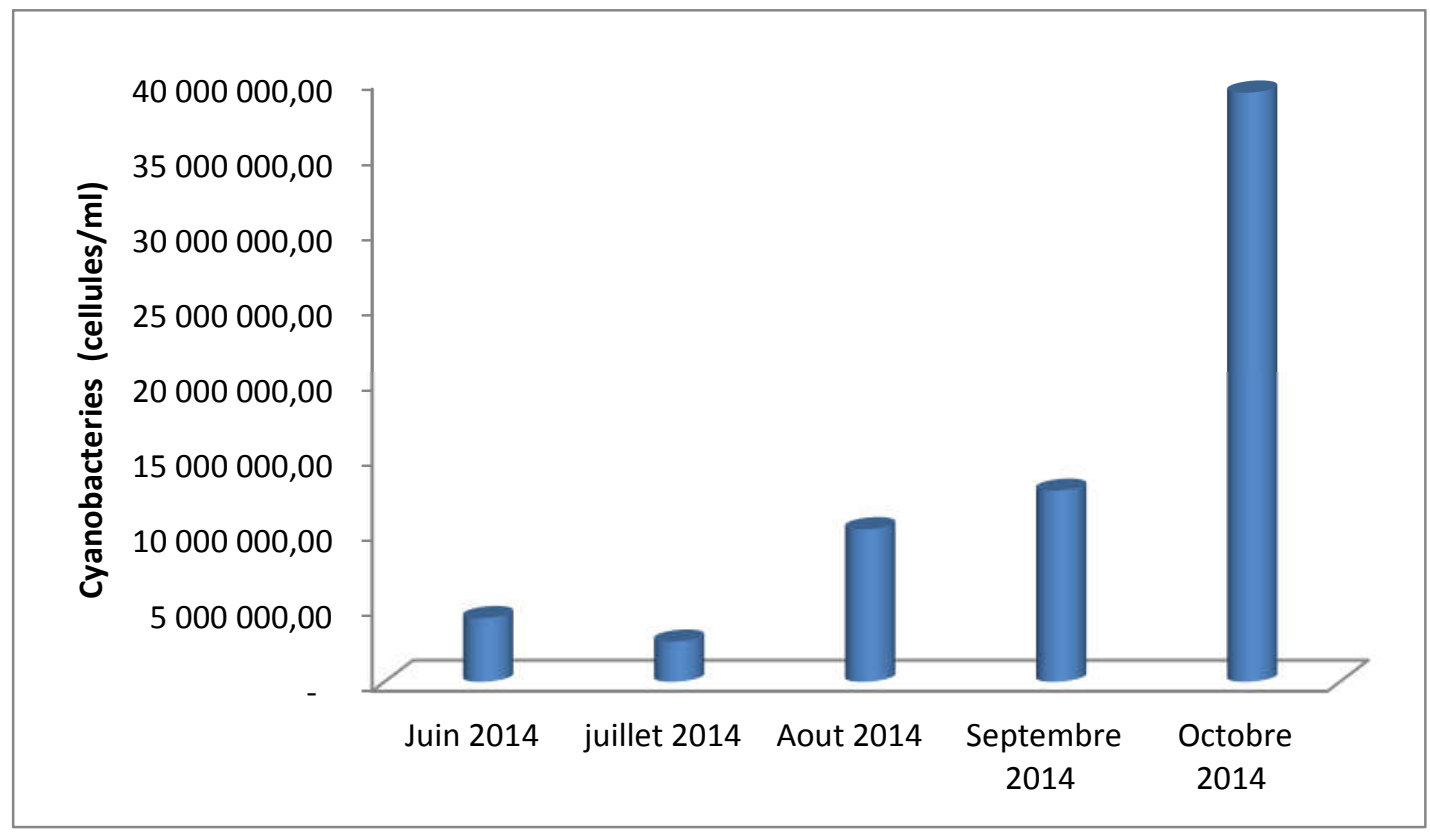

Figure 1: Evolution du nombre de Cyanobactéries suivant les mois et les sites d'échantillonnage de juin à octobre 2014 . 


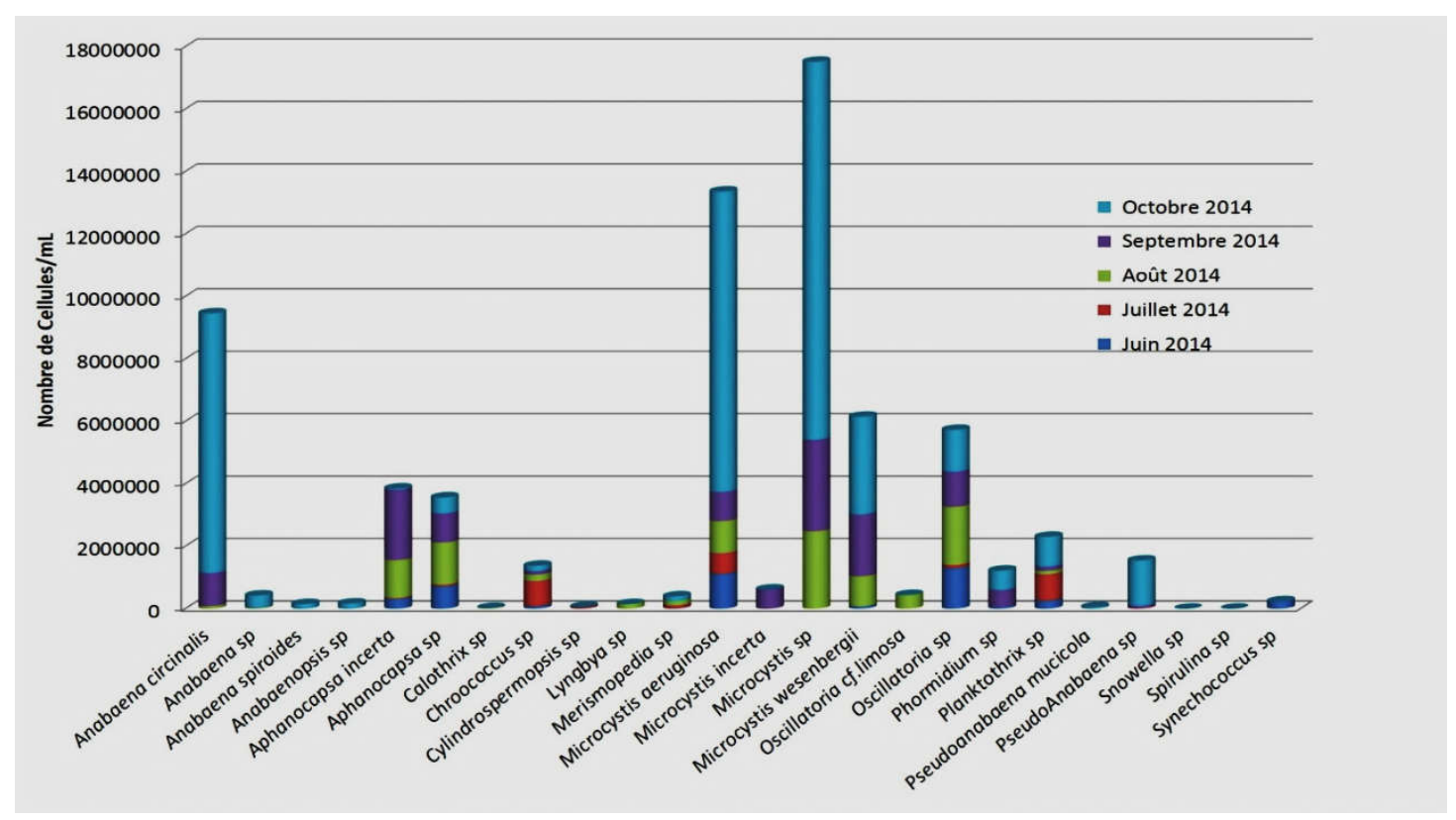

Figure 2: Evolution temporelle des différentes espèces de cyanobactéries sur les sites d'Adzopé, d'Agboville et d'Aghien.

Tableau 2:Proportion des genres de cyanobactéries dans la Lagune Aghien.

\begin{tabular}{|c|c|c|c|c|c|c|}
\hline Lagune Aghien & $\begin{array}{l}\text { Juin } \\
\text { (N) }\end{array}$ & $\begin{array}{l}\text { Juillet } \\
\text { (N) }\end{array}$ & $\begin{array}{l}\text { Aout } \\
\text { (N) }\end{array}$ & $\begin{array}{l}\text { Septembre } \\
\text { (N) }\end{array}$ & $\begin{array}{l}\text { Octobre } \\
\text { (N) }\end{array}$ & $\begin{array}{l}\text { Total } \\
\text { N(\%) }\end{array}$ \\
\hline Anabaena & - & - & 11,75 & 106,71 & 873,75 & $992,21(\mathbf{1 6 \%})$ \\
\hline Anabaenopsis & 0,13 & - & - & - & 11,50 & $11,63(\mathbf{0 \%})$ \\
\hline Aphanocapsa & 101,13 & 8,13 & 257,21 & 317,90 & 42,25 & 726,61 (12\%) \\
\hline Calothrix & - & - & 2,08 & - & - & $2,08(\mathbf{0 \%})$ \\
\hline Chroococcus & 7,75 & 6,50 & 20,50 & 11,55 & 15,63 & $61,92(\mathbf{1 \%})$ \\
\hline Cylindrospermopsis & 0,10 & 5,25 & - & - & - & $5,35(\mathbf{0 \%})$ \\
\hline Lyngbya & - & - & 15,00 & - & - & $15,00(\mathbf{0 \%})$ \\
\hline Merismopedia & 0,50 & - & 13,61 & - & 15,00 & 29,11 (0\%) \\
\hline Microcystis & 120,38 & 61,00 & 448,25 & 644,84 & 2390,25 & $3664,72(\mathbf{5 8 \%})$ \\
\hline Oscillatoria & 33,38 & 5,00 & 192,50 & 112,23 & 28,75 & $371,86(\mathbf{6 \%})$ \\
\hline Phormidium & 7,00 & - & - & 53,13 & 61,88 & $122,00(\mathbf{2 \%})$ \\
\hline Planktothrix & 26,25 & 6,38 & 11,75 & 13,25 & 95,25 & $152,88(\mathbf{2 \%})$ \\
\hline Pseudoanabaena & - & 2,50 & - & 7,02 & 104,00 & $113,52(\mathbf{2 \%})$ \\
\hline Snowella & - & - & - & - & 0,22 & $0,22(\mathbf{0 \%})$ \\
\hline Spirulina & - & - & - & 0,13 & - & $0,13 \mathbf{( 0 \% )}$ \\
\hline Synechococcus & 23,88 & - & - & - & - & $23,88(\mathbf{0 \%})$ \\
\hline
\end{tabular}

$\mathrm{N}=$ nombre de cellules $\mathrm{X} 10^{4} / \mathrm{ml} ;(-)=$ non définie. 
Tableau 3: Distribution des genres de cyanobactéries dans la Retenue d'eau d'Adzopé (2014).

\begin{tabular}{|c|c|c|c|c|c|c|}
\hline Adzopé & $\begin{array}{l}\text { Juin } \\
\text { (N) }\end{array}$ & $\begin{array}{l}\text { Juillet } \\
\text { (N) }\end{array}$ & $\begin{array}{l}\text { Aout } \\
\text { (N) }\end{array}$ & $\begin{array}{l}\text { Septembre } \\
\text { (N) }\end{array}$ & $\begin{array}{l}\text { Octobre } \\
\text { (N) }\end{array}$ & $\begin{array}{l}\text { Total } \\
\text { N (\%) }\end{array}$ \\
\hline Anabaena & - & - & - & - & 5,00 & $5,00(1 \%)$ \\
\hline Anabaenopsis & - & - & - & - & 5,00 & $5,00(\mathbf{1 \%})$ \\
\hline Aphanocapsa & - & - & - & - & 12,50 & $12,50(\mathbf{3 \%})$ \\
\hline Calothrix & - & 0,33 & - & - & - & $0,33(\mathbf{0} \%)$ \\
\hline Chroococcus & 1,00 & 73,75 & - & - & 1,00 & 75,75 (15\%) \\
\hline Cylindrospermopsis & 0,40 & - & - & - & - & $0,40(\mathbf{0 \%})$ \\
\hline Merismopedia & 0,50 & 10,00 & - & - & - & $10,50(\mathbf{2 \%})$ \\
\hline Microcystis & - & 5,00 & - & - & 95,13 & $100,13(\mathbf{2 0} \%)$ \\
\hline Oscillatoria & 97,15 & 4,75 & 25,80 & - & 30,00 & $157,70(\mathbf{3 2} \%)$ \\
\hline Planktothrix & - & 77,50 & - & - & 0,00 & $77,50(\mathbf{1 6 \%})$ \\
\hline Pseudoanabaena & - & - & - & - & 45,00 & 45,00 (9\%) \\
\hline
\end{tabular}

Tableau 4: Proportion des genres de cyanobactéries dans le Seuil d'Agboville.

\begin{tabular}{lcccccc}
\hline Agboville & $\begin{array}{c}\text { Juin } \\
(\mathbf{N})\end{array}$ & $\begin{array}{c}\text { Juillet } \\
(\mathbf{N})\end{array}$ & $\begin{array}{c}\text { Aout } \\
(\mathbf{N})\end{array}$ & $\begin{array}{c}\text { Septembre } \\
(\mathbf{N})\end{array}$ & $\begin{array}{c}\text { Octobre } \\
(\mathbf{N})\end{array}$ & $\begin{array}{c}\text { Total } \\
\mathbf{N}(\mathbf{\%})\end{array}$ \\
\hline Anabaena & - & - & - & - & 6,25 & $6,25(\mathbf{6 \%})$ \\
Aphanocapsa & 2,50 & - & - & - & - & $2,50(\mathbf{3 \%})$ \\
Oscillatoria & - & - & 12,50 & - & 75,00 & $87,50(\mathbf{9 0 \%})$ \\
Pseudoanabaena & - & - & - & - & 1,00 & $1,00(\mathbf{1 \%})$ \\
& & & & & &
\end{tabular}




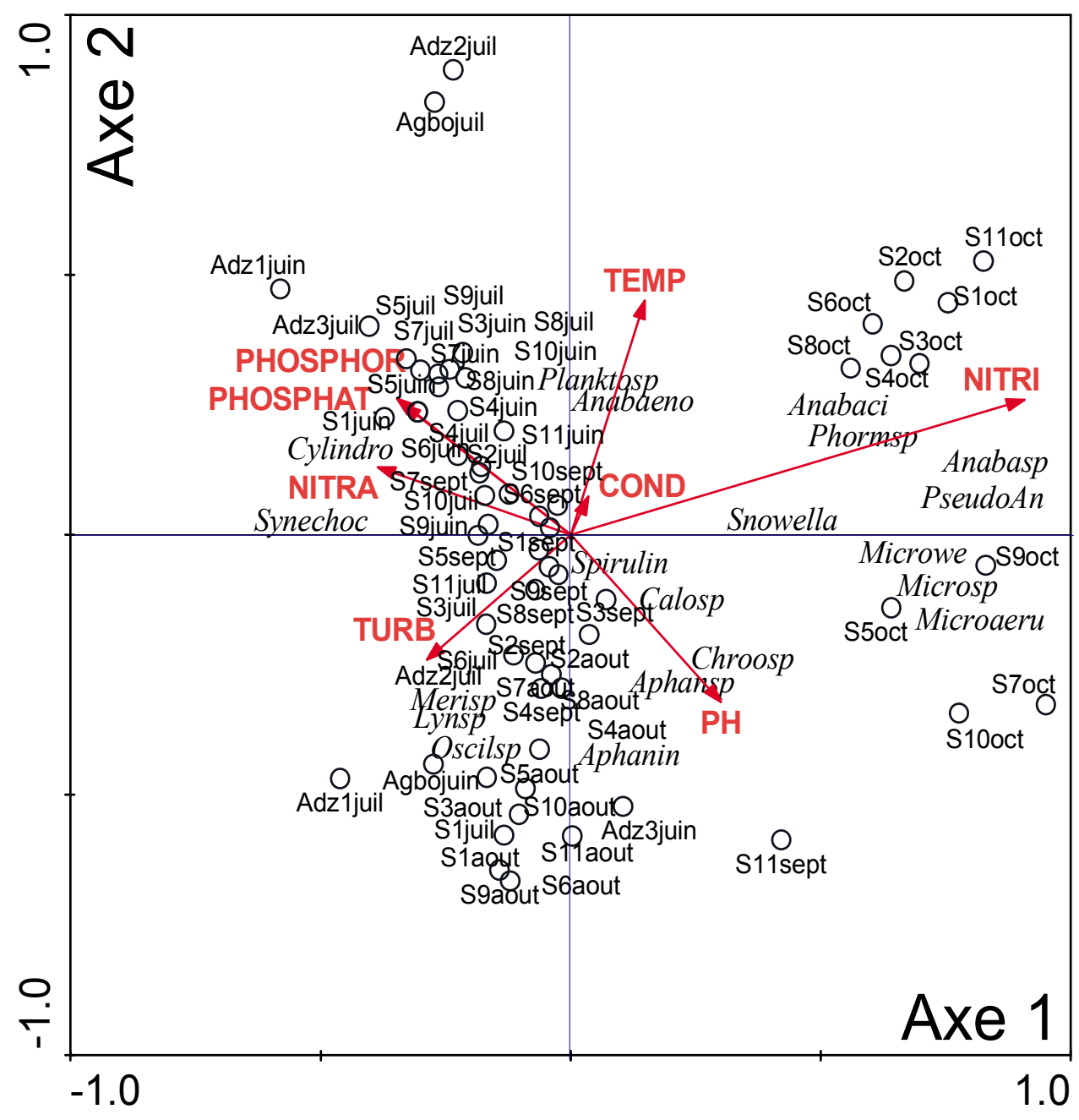

Figure 3:Paramètres environnementaux influençant la distribution des cyanobactéries

\section{DISCUSSION}

La présente étude a été entreprise afin de faire l'inventaire des espèces de cyanobactéries en général et de déterminer les genres potentiellement toxiques dans des plans d'eau de Côte d'Ivoire en particulier, par des techniques microscopiques. Un nombre assez important de genres de cyanobactéries différentes a été identifié sur les trois (3) sites échantillonnés à savoir la Lagune Aghien, la retenue d'Adzopé, et le seuil d'Agboville. En effet, 15 genres de cyanobactéries et 20 taxons ont été identifiés.
Ces proportions sont proches de celles rapportées par Groga et al. (2012) à Taabo, Salla et al. (2011) sur la Mé et Boubo, et Adon et al. (2011) à Adzopé. La présence des genres Microcystis Anabeana et Planktothrix dans la Lagune Aghien a confirmé les résultats de Ngohesse et al. (2007) et de Humbert et al (2012). Le nombre de taxons retrouvés au cours de ce travail (20) est inférieur de celui au cours des travaux exclusivement réalisés sur les cyanobactéries en Côte d'Ivoire, (29) Bourrelly (1961) et (27) Salla et al. (2011). Ces nombres de taxons 
sont inférieur de ceux retrouvés dans d'autres pays, 29 au Sénégal et 23 au Burkina Faso (Thomazeau et al., 2006).

Cependant, il est à noter que si les genres de cyanobactéries isolés diffèrent qualitativement suivant les sites, certains sont constamment retrouvés notamment Aphanocapsa, Microcystis et Anabeana. Parmi les genres de cyanobactéries étudiées certaines sont potentiellement toxiques. Il a été retrouvé neuf (9) genres dont six (6) potentiellement sécrétrices de microcystines. Ce nombre est du même ordre que celui de toutes les études antérieures en Côte d'Ivoire. Ainsi Groga et al. (2012) ont trouvé 5 genres potentiellement toxiques quand Adon et al. (2011) et Salla et al. (2012) en identifiaient respectivement 4 et 7 . Le genre Microcystis n'a pas été retrouvé à Agboville.

Les cyanobactéries ont connu une évolution qualitative et quantitative au cours de ce travail. En effet, le nombre de genres et les effectifs ont considérablement augmenté au fil $\mathrm{du}$ temps. Les principaux genres potentiellement toxiques que sont Planktothrix, Anabaena, Oscillatoria et Microcystis (Haider et al., 2003 ; Glowacka et al., 2011) ont été observés. Globalement, Microcystis a été le plus présent et dominait majoritairement les cyanobactéries. Cependant, au niveau des sites, si Microcystis a été majoritaire sur la Lagune Aghien, Oscillatoria a été le plus présent sur les sites d'Adzopé et d'Agboville. Cette dominance des Microcystis pourrait s'expliquer par le nombre de stations de prélèvement plus élevé sur la Lagune Aghien d'une part et d'autre part par le fait que le genre Microcystis est le plus répandu à travers le monde surtout lors des efflorescences comme l'ont souligné Oudra et al. (2002) au Maroc. En effet les blooms à Cyanobactéries observé à Adzopé et Aghien étaient dominés par ce genre. Ces genres sont considérés comme étant les plus toxiques ( $\mathrm{Li}$ et al., 2011; Mbwaka et al.,
2012). Ce qui met en évidence le caractère potentiellement dangereux de ces proliférations dans les eaux étudiés.

L'évolution des cyanobactéries au cours de la présente étude a atteint son niveau maximum au mois d'octobre. Elle pourrait s'expliquer par le nombre de taxons apparaissant au fil du temps. Le genre Anabaena qui était absent au mois de juin est par la suite apparu et s'est développé assez rapidement. Ce phénomène a également été observé dans des lacs réservoirs au Maroc (Oudra et al., 2002; Khlil et al., 2011) avec une variation continue suivant les années.

Les résultats montrent le caractère eutrophe à hypereutrophe des sites étudiés (CCME, 2004 ; MDDELCC, 2015 ; OBVS, 2016). Ce caractère, pourrait s'expliquer par la pression anthropique exercée sur leurs bassins versants. En effet, la Lagune Aghien est entourée de très nombreuses plantations et unités de production d'huile de palme. C'est le lieu également de réalisation de nombreuses activités ménagères des villages la bordant (Ngohessé, 2007). Cette lagune est alimentée par la Rivière Djibi et le Fleuve Mé qui draine un bassin versant de $4000 \mathrm{~m}^{2}$ en plus d'un éclairage accentué.

La retenue d'Adzopé située en pleine agglomération est entourée d'habitations et est caractérisée par un déversement d'eaux usées et d'effluents de nombreuses industries de bois. Les cyanobactéries étaient donc dans des environnements avec des éléments nutritifs abondants favorables à leur développement. En effet, Woch (2006) a rapporté que l'apport de phosphore et/ou d'azote dans des milieux naturellement pauvres en ces nutriments favorisait le développement des cyanobactéries. L'action de ces nutriments est démontrée par les fortes corrélations qui les associaient aux cyanobactéries. L'augmentation des cyanobactéries à partir du mois d'Août correspondant à la fin de la grande saison des pluies a continué en septembre et Octobre qui correspondent à la petite saison des pluies. L'augmentation du genre Anabaena a suivi celle du genre 
Microcystis. Cette dominance conjointe avec une supériorité pour Microcystis a été retrouvé aussi par Te et al. (2011) au Singapour.

L'analyse de redondance et le test de Pearson ont démontré le rôle capital du nitrite, $\mathrm{du}$ nitrate, du phosphore, du $\mathrm{pH}$, de la température et de la turbidité sur la distribution des cyanobactéries. Ce test a montré une forte corrélation statistiquement significative entre la présence de cyanobactéries et le nitrite $(\mathrm{r}: 0,99 ; \mathrm{p}<0,017)$. Il a été observé une forte corrélation positive entre les concentrations de phosphore $(\mathrm{r}$ : $0,99 ; \mathrm{p}<0,02)$ et de phosphate $(\mathrm{r}: 0,99$; $\mathrm{p}<0,01)$ et l'abondance des cyanobactéries. Cette forte association est également retrouvée au Brésil (Costa et al., 2014). Le rôle de la température dans les proliférations mis en évidence dans cette étude est soutenu par Paërl et al. (2008) et Te et al. (2011). De plus, Lürling et al. (2013) observait que l'action simultanée du phosphore et de la température favoriserait le développement des cyanobactéries. La domination du genre Microcystis dans cette étude pourrait être attribuée à ces effets dans nos régions. En effet selon Imai et al. (2009) les cyanobactéries du genre Microcystis prolifère bien dans les hautes températures.

Les tests d'association des paramètres a montré une corrélation positive entre le nombre de genres de cyanobactéries et la turbidité $(\mathrm{r}: 0,67 ; \mathrm{p}<0,02)$, confirmant ainsi l'observation de l'analyse de redondance sur la Lagune Aghien. Delarras et al. (2010) montrent en effet que la turbidité est l'un des principaux facteurs physiques favorisant la prolifération des cyanobactéries. Costa et al. (2014), indique que la turbidité participerait effectivement à la prolifération des cyanobactéries mais serait une conséquence de l'hypereutrophisation des plans d'eau.

\section{Conclusion}

La prolifération des cyanobactéries dans les plans d'eau étudiés est sous le contrôle de l'hypereutrophisation à travers la concentration en nutriment mais aussi des paramètres physiques. Les formes dissoutes $\mathrm{du}$ phosphore et notamment le phosphate agiraient en association avec la température. Cette prolifération des cyanobactéries se fait surtout à travers la prolifération des Microcystis et des Anabaena principalement.

\section{CONFLIT D'INTERETS}

Les auteurs ne déclarent aucun conflit d'intérêts.

\section{CONTRIBUTIONS DES AUTEURS}

$\mathrm{C}-\mathrm{KJ}$ a écrit le protocole, conduit les travaux, écrit l'article; SMK a réalisé les analyses statistiques ; NEJE a réalisé les identifications des cyanobactéries; YK a participé aux missions de terrain et aux analyses physicochimiques; $\mathrm{AL}$ a participé aux missions de collecte et aux analyses physico-chimiques; DAJ a corrigé le protocole, supervisé les analyses chimiques; DM a supervisé les travaux et corrigé l'article.

\section{REMERCIEMENTS}

Les auteurs adressent leurs sincères remerciements, à l'Institut Pasteur de Côte d'Ivoire, au Laboratoire d'Ecologie et de Biologie Aquatique de l'Université Nangui Abrogoua, à l'Office National de l'Eau Potable de Côte d'Ivoire et à tous ceux qui ont contribué à la réalisation de ce travail. Merci aussi au Ministère des Eaux et Forêts et à l'Agence Française de Développement.

\section{REFERENCES}

Adon MP, Ouattara A, Gourene G. 2011. Seasonal variation in the diversity and abundance of phytoplankton in a small African tropical reservoir. Afr. J. Microbiol. Res., 5(18): 2616-2026. DOI: 10.5897/AJMR11.196

Adon MP. 2013. Variations spatiale et saisonnière du phytoplancton de la retenue d'eau d'adzopé (Côted'Ivoire) : 
composition, structure, biomasse et production primaire. Thèse Unique, Université Nangui Abrogoua, Abidjan, Côte d'Ivoire.

Azevedo L, Vilarinho L, Teles EL, Amorim A. 2002. Ornithine transcarbamylase deficiency: a novel splice site mutation in a family with meiotic recombination and a new useful SNP for diagnosis. Mol. Genet. Metab., 76(1): 68-70.

Bertrand F, Dunand A, Fosse J, Fradin N, Liger D, Gérard Keck. 2004. Les intoxications par les cyanobactéries. Le Point Vétérinaire, 250 : 46-50.

Bourrelly P. 1961. Cyanophycées de la Côte d'Ivoire. Schweiz Z Hydrol., 23(21): 209-210.

Briand E. 2008. Contribution à la compréhension du déterminisme de la mise en place des proliférations de cyanobactéries et de leur production de toxines. Thèse de Doctorat, Muséum national d'Histoire Naturelle, Paris, France.

Carmichael WW. 2001. Health effects of toxin-producing cyanobacteria: the CyanoHABs. Hum. Ecol. Risk Assess, 7: 1393-1407.

Chorus I, Falconer IR, Salas HJ, Bartram J. 2000-. Health risks caused by freshwater cyanobacteria in recreational waters. $J$ Toxicol. Environ. Health, Part B: Critical Reviews, 3(4): 323-347.

Conseil canadien des ministres de l'environnement (CCME). 2004. Recommandations canadiennes pour la protection de la vie aquatique : le phosphore : cadre canadien d'orientation pour la gestion des réseaux hydrographiques. Dans : Recommandations canadiennes pour la qualité de l'environnement, CCME, Winnipeg

Costa SM, Appel E, Macedo CF, Huszar VLM. 2014. Low water quality in tropical fishponds in southeastern Brazil. An Acad Bras. Cienc., 86(3): 1181-1195. http://dx.doi.org/10.1590/000137652014 20130092

Delarras C, Trébaol B, Durand J. 2010. Surveillance Sanitaire et Microbiologique des Eaux: Réglementation, Micro-organismes, Prélèvements, Analyses (2e Édn). Edition Lavoisier/ Tec et Doc: Paris, France.

Garrity GM, Boone DR, Castenholz RW. 2001. Bergey's Manual of Systematic Bacteriology: The Archea and the Deeply Branching and Phototrophic Bacteria (2nd edn, Vol. 1). Springer Verlag: New York; 427-446.

Głowacka J, Szefel-Markowska M, Waleron M, Łojkowska E, Waleron K. 2011. Detection and identification of potentially toxic cyanobacteria in Polish water bodies. Acta Biochim. Pol., 58(3): 321-333.

Groga N. 2012.- Structure, fonctionnement et dynamique du phytoplancton dans le lac de Taabo (Côte d'Ivoire). Thèse de Doctorat, Université de Toulouse, France.

Fosso-Kankeu E, Jagals P, Du Preez H. 2008. Exposure of rural households to toxic cyanobacteria in container-stored water. Water SA., 34(5): 631-636. Available on website http://www.wrc.org.za.

Haider S, Naithani V, Viswanathan PN, Kakkar P. 2003. Cyanobactérial toxins: a growing environmental concern. Chemosphere, 52: 1-21.

Humbert JF. 2012.- Rapport sur la mission : Lagune Aghien, Abidjan, Côte d'Ivoire.

Imai H, Chang K-H, Kusaba M, Nakano S-I. 2009. Temperature-dependent dominance of Microcystis (Cyanophyceae) species: M. aeruginosa and M. wesenbergii. Journal of Plankton Research, 31: 171-178. 
John DM, Whitton BA, Brook AJ. 2003. The Freshwater Algal Flora of the British Isles, An Identification Guide to Freshwater and Terrestrial Algae. Cambridge University Press: Cambridge. Jöhnk KD, Huisman J, Sharples J, Sommeijer B, Visser PM, Stroom JM. 2008. Summer heatwaves promote blooms of harmful cyanobacteria. Glob. Change Biol., 14(3): 495-512. DOI: 10.1111/j.1365-2486.2007.01510.x

Khlil N, Zidane F, Naamane A, Loufti M, Blagen M. 2011. Contribution à l'évaluation des traces de microcystines dissoutes et intracellulaire dans les eaux marocaines brutes et traitées. Rev. Sci. Eau, 24(4) : 355-368.

Komárek J, Anagnostidis K. 2005. Cyanoprokaryota-2. Teil/ 2nd Part : Oscillatoriales. In Süßwasserflora von Mitteleuropa 19/2, Büdel B, Gärtner G, Krenitz L, Schagerl M (Éds.). Elsevier: Heidelberg.

Komárek J, Anagnostidis K. 1998. Cyanoprokaryota-1. Teil : Chroococcales. In Süßwasserflora von Mitteleuropa 19/1, Ettl H, Gärtner G, Heynig H, Mollenhauer D (Éds.). Gustav Fischer: Jena.

Leitao M, Couté A. 2005. Guide pratique des cyanobactéries du Grand Ouest de la France. Edition: Agence de l'Eau Seine Normandie, Nanterre, France.

Li L, Jia R, Liu Y, Zhang H. 2011. Detection of Microcystin-Producing Cyanobacteria in a reservoir by whole cell quantitative PCR. Procedia Environ. Sci., 10: 22722279.

http://dx.doi.org/10.1016/j.proenv.2011. 09.355

Lürling M, Eshetu F, Faassen E, Kosten S, Huszar VLM. 2013. Comparison of cyanobacterial and green algal growth rates at different temperatures.
Freshwater Biol., 58: 552-559. DOI: 10.1111/j.1365-2427.2012.02866.x

Mbukwa EA, Msagati TA, Mamba BB. 2012.Quantitative Variations of Intracellular Microcystin-LR, -RR and -YR in Samples Collected from Four Locations in Hartbeespoort Dam in North West Province (South Africa) During the 2010/2011 Summer Season. Int. J. Environ. Res. Public Health, 9(10): 3484-3505. doi:10.3390/ijerph9103484

Ministère du Développement Durable, de l'Environnement et de la Lutte contre les Changements Climatiques (MDDELCC). 2015. Le Réseau de surveillance volontaire des lacs: Les méthodes Qu'est-ce que l'eutrophisation?, En ligne:

http://www.mddelcc.gouv.qc.ca/eau/rsvl/ methodes.htm, consulté le 1er octobre 2015

N'Gohesse-Kouassi E. 2007.- Caractérisation phytoplanctonique et bactériologique des eaux de la Lagune Aghien (Cote d'Ivoire). Mémoire de DEA, Université Abobo-Adjamé, Abidjan, Côte d'Ivoire.

Ni WM, Zhang JY, Ding TD, Stevenson RJ, Zhu Y.M. 2012.- Environmental factors regulating cyanobacteria dominance and microcystin production in a subtropical lake within the Taihu watershed, China. $J$ Zhejiang Univ-Sci A (Appl Phys \& Eng), 13(4): 311-322.

Niamien-Ebrottie JE, Bhattacharyya S, Deep PR, Nayak B. 2015. Cyanobacteria and cyanotoxins in the World: Review. International Journal of Applied Research, 1(8): 563-569.

Organisme de Bassin Versant du Saguenay (OBVS). 2016. Portrait du bassin versant du lac Déchêne-2015. Rapport technique préparé pour la Municipalité de Larouche, Ville de Saguenay, 29 pages et 1 annexe.

Ouattara A. 2000. Premières données systématiques et écologiques du 
phytoplancton du lac d'Ayamé (Côte d'Ivoire). Thèse de doctorat, Katholieke Universiteit Leuven, Belgique : $207 \mathrm{p}$.

Oudra B, Loudiki M, Sabour B, Sbiyyaa B, Vasconcelos V. 2002. Etude des blooms toxiques à Cyanobactéries dans trois lacs réservoirs du Maroc: Résultats préliminaires. Rev. Sci. Eau, 15(1): 301303. DOI : 10.7202/705454ar

Ouellette AJA, Wilhelm SW. 2003.- Toxic cyanobacteria: the evolving molecular toolbox. Front Ecol. Environ., 1(7): 359-366.

Paerl HW, Huisman J. 2008. Blooms like it hot. Science, 320: 57-58. http://dx.doi.org/10.1126/science.115539 8

Robin J, Cravedi JP, Hillenweck A, Deshayes C, Vallod D. 2006. Off flavor characterization and origin in French trout farming. Aquacult., 260: 128-138.

Salla M, Kouhete PD, Koffi SO, Traore D. 2011. Cyanobactéries des rivières Boubo et Mé dans le Sud côtier de la Côte d'Ivoire. Int. J. Biol. Chem. Sci., 5(4): 1365-1373.

DOI: http://dx.doi.org/10.4314/ijbcs.v5i4.3
Te SH, Gin KY-H. 2011. The dynamics of cyanobacteria and microcystin production in a tropical reservoir of Singapore. Harmful Algae, 10(3): 319329.

http://dx.doi.org/10.1016/j.hal.2010.11.0 06

Thomazeau S. 2006. « Diversité phylogénétique et toxinique des cyanobactéries du Sénégal et du Burkina Faso ». Mémoire de Master 2, Université Paris 6, Mention Sciences de l'Univers, Environnement, Ecologie, Paris, France.

Willame R, Jurczak T, Iffly J-F, Kull T, Meriluoto J, Hoffman L. 2005. Distribution of hepatotoxic cyanobacterial blooms in Belgium and Luxembourg. Hydrobiologia, 551: 99117.

Woch M. 2006. Suivi au Phyto-PAM d'expériences mixtes d'enrichissements et d'opacifications des communautés phytoplanctoniques du réservoir de Loumbila (Burkina Faso). Licence Génie de l'Environnement. Université Paris 7 Denis Diderot, Paris, France. 